\title{
Frequency and topography of small cerebrovascular lesions in vascular and in mixed dementia: a post-mortem 7-tesla magnetic resonance imaging study with neuropathological correlates
}

\author{
Jacques De Reuck, Florent Auger, Nicolas Durieux, Vincent Deramecourt, Claude-Alain Maurage, \\ Charlotte Cordonnier, Florence Pasquier, Didier Leys, Regis Bordet \\ Centre Hospitalier Régional Universitaire de Lille (CHRU), Lille, France
}

\begin{abstract}
Introduction: Mixed dementia (MixD) refers to a combination of definite Alzheimer's disease (AD) and vascular encephalopathy. The existence of a "pure" type of vascular dementia (VaD) is controversial. There is a need to find magnetic resonance imaging (MRI) characteristics allowing the distinction between VaD and MixD. The present post-mortem 7.0-tesla MRI compares the frequency or severity and the topography of the small cerebrovascular lesions in brains of patients with VaD and with MixD.

Material and methods: Based on neuropathological criteria, 14 brains were classified as VaD, 24 as MixD and 11 as controls. Three coronal sections of a cerebral hemisphere and a horizontal section of a cerebellar hemisphere underwent T2 and T2* 7.0-tesla MRI examination. The mean values and topographic distribution of white matter changes (WMCs), lacunar infarcts (LIS), cortical microbleeds (CoMBs) and cortical microinfarcts (CoM/s) were determined and compared between the different groups.

Results: Compared to the controls, both VaD and MixD brains had significantly more severe WMCs and increased numbers of CoMBs and CoMIs. Lacunar infarcts predominated only in the VaD cases. On mutual comparison of VaD and MixD brains, COMBs and COMIs predominated in the frontal lobe and the cerebellum of VaD, while were mainly present in the occipital lobe of MixD. White matter changes predominated in the temporal lobe of MixD cases. Lacunar infarcts were significantly increased in the corona radiata and putamen of VaD patients.

Conclusions: The present post-mortem MRI study shows clear differences in the distribution and the types of cerebrovascular lesions on high-field MRI, confirming that VaD and MixD are different diseases.
\end{abstract}

Key words: post-mortem 7.0-tesla MRI, vascular dementia, mixed dementia, topographic distribution of small cerebrovascular lesions, cortical microbleeds, cortical microinfarcts, white matter changes, lacunar infarcts.

Communicating author:

Prof. Jacques De Reuck, CHRU Lille, EA 1046, Leopold II Laan 96, 9000 Ghent, Belgium, phone: 3292218844,3292215668 ,

e-mail: dereuck.j@gmail.com 


\section{Introduction}

Mixed dementia (MixD) refers to a combination of definite Alzheimer's disease (AD) and vascular encephalopathy. The distinction between MixD and "pure" vascular dementia is controversial [15,23]. In demented patients vascular lesions on structural magnetic resonance imaging (MRI) are often misdiagnosed as probable vascular dementia ( $\mathrm{VaD})$ as compared to autopsy-confirmed diagnosis [20]. Also the utility of their detection for the individual diagnosis of $\mathrm{VaD}$ or MixD is limited [28]. Major vascular lesions differ between $\mathrm{VaD}$ and MixD [17].

An important obstacle in the standardization of diagnosis is the fact that vascular brain lesions are a large group comprising heterogeneous changes that have different pathogenesis [14]. Our previous post-mortem study showed that the lesion pattern in $\mathrm{VaD}$ and MixD is different: while the global severity of the white matter changes is more or less similar, lacunes in the corona radiata predominate in the former and cerebral amyloid angiopathy-related lesions in the latter [9].

So there is a need to quantify and determine the topography of the different types of cerebrovascular lesions in $\mathrm{VaD}$ and MixD. Post-mortem MRI is an additional complement of the neuropathological assessment of these lesions [21]. 7-tesla MRI is the most suitable technique to detect small cerebrovascular lesions in post-mortem brains [26].

The present post-mortem 7.0-tesla MRI study with neuropathological correlates investigates whether there are differences in severity and topography of small cerebrovascular lesions between $\mathrm{VaD}$ and MixD brains in order to find neuroimaging criteria that allow the distinction between both disease entities.

\section{Material and methods}

Out of a series of 162 consecutive autopsied patients, followed up at the Lille University Hospital, who underwent a MRI examination, according to the neuropathological criteria [2,4], 14 with a diagnosis of $\mathrm{VaD}, 24$ of MixD and 11 control brains, without a history of dementia or stroke, were selected.

They all underwent a post-mortem 7.0-tesla MRI examination of 3 coronal sections of a cerebral hemisphere and one horizontal section of a cerebellar hemisphere, followed by an extensive histological examination of the brain samples.
Previously obtained informed consent of the patients or from the nearest family allowed an autopsy for diagnostic and scientific purposes. The brain tissue samples were acquired from the Lille Neuro-Bank of the Lille University that is a part of the "Centres des Resources Biologiques" and acts as an institutional review board.

One fresh cerebral hemisphere was frozen for biochemical examination. The remaining hemisphere, the brainstem, and most of the cerebellum were fixed in formalin for three weeks.

\section{Neuropathological examination}

The disease diagnosis was made according a standard procedure and examination of a large number of samples. In addition to the detection of macroscopic visible lesions such as haematomas, territorial and lacunar infarcts (LIS), a whole coronal section of a cerebral hemisphere, at the level of the mammillary body and a horizontal section of a cerebellar hemisphere were taken for the semi-quantitative evaluation of the small cerebrovascular lesions such as white matter changes (WMCs), cortical microbleeds (COMBs), and cortical microinfarcts (CoMIs).

The mean values for WMCs were the average of the ranking scores: no change (RO), a few isolated (R1), frequent scattered in the corona radiata (R2) and forming confluent lesions (R3) of myelin and axonal loss. For the other cerebrovascular lesions, their mean values corresponded to their percentage number [6].

The diagnosis of cerebral angiopathy (CAA) was made when a majority of $\beta$-amyloid stained vessels were present in at least three of the four examined samples and as not-CAA, $\beta$ when absent or scarce, in case of a few stained vessels in one or two slides [13].

\section{Magnetic resonance imaging examination}

Three coronal sections of a cerebral hemisphere were submitted to T2 and T2* MRI: a frontal one at the level of the head of the caudate nucleus, a central one at the level of the mammillary body and one at the level of the parietal and occipital lobes. In addition, one horizontal section of a cerebellar hemisphere was also examined.

A 7.0-tesla MRI Bruker BioSpin SA with an issuer-receiver cylinder coil of $72 \mathrm{~mm}$ inner diameter 
(Ettlingen, Germany) was used, according to a previous described method [7].

The ranking scores of severity of the WMCs were evaluated separately in the different brain sections as done on the neuropathological section. Lacunar infarcts were defined as small-rounded lesions with a diameter between 3 and $15 \mathrm{~mm}$ in the corona radiata, internal capsule, caudate nucleus, putamen, globus pallidus, thalamus and cerebellar white matter [27]. The number and the location of the small cerebrovascular lesions were determined by consensus evaluation of three observers (JDR, FA, ND), blinded to the neuropathological diagnosis. The inter-rater reliability resulted in an interclass correlation coefficient of 0.82 .

\section{Statistical analysis}

Univariate comparisons of unpaired groups were performed with the Fisher's exact test for categorical data. The non-parametric Mann-Whitney U-test was used to compare continuous variables. The significance level, two-tailed, was set at $\leq 0.01$ for significant and $\leq 0.001$ for highly significant. Values set at $\leq 0.05$ but more than $>0.01$ were considered as marginal significant and not included as relevant due to the relative small sample sizes.

\section{Results}

The average age at death was not significantly different between the groups: $75( \pm 10)$ years in the VaD patients, $76( \pm 11)$ in the MixD and $71( \pm 9)$ in the control group $(p=0.16)$. Also the gender distribution was similar with $80 \%$ males in $\mathrm{VaD}, 54 \%$ in MixD and $73 \%$ in the control groups, respectively $(p=0.62)$.

Arterial hypertension and the use of antithrombotic agents were the only more frequently found clinical vascular risk factors in the $\mathrm{VaD}$ and MixD patients compared to the controls (Table I).

On neuropathological examination, the semi- quantitative evaluation of the degree of severity showed a significant increase in WMCs and higher incidence of CoMls and CoMBs in VaD as MixD compared to controls. On the other hand, LIs and territorial infarcts were only more frequent in the $\mathrm{VaD}$ group, while CAA related lesions were more observed in the MixD group (Table II).

Table I. Comparison of vascular risk factors between normal controls (C) and patients with vascular dementia (VaD) and mixed dementia (MixD)

\begin{tabular}{|lccc|}
\hline Vascular risk factors & $\mathrm{C}$ & VaD & MixD \\
\cline { 2 - 4 } & $(n=11)$ & $(n=14)$ & $(n=24)$ \\
\hline Arterial hypertension & $18 \%$ & $86 \%^{* *}$ & $75 \%^{*}$ \\
\hline Diabetes & $0 \%$ & $43 \%$ & $33 \%$ \\
\hline Hypercholesterolemia & $27 \%$ & $50 \%$ & $46 \%$ \\
\hline Smoking & $18 \%$ & $21 \%$ & $13 \%$ \\
\hline Antithrombotic use & $18 \%$ & $93 \%^{* *}$ & $83 \%^{* *}$ \\
\hline
\end{tabular}

${ }^{*} p \leq 0.01 ;{ }^{* *} p \leq 0.001$

Table II. Mean values (standard deviations) of the neuropathological lesions in normal controls (C) compared to those in vascular dementia (VaD) and mixed dementia (MixD) brains

\begin{tabular}{|llcc|}
\hline \multicolumn{1}{|c}{ Cerebrovascular lesions } & \multicolumn{1}{c|}{ C } & VaD & MixD \\
\cline { 2 - 4 } & $(n=11)$ & $(n=14)$ & $(n=24)$ \\
\hline White matter changes & $0.4(0.7)$ & $1.7(1.3)^{\star}$ & $1.4(1.2)^{\star}$ \\
\hline Cerebral amyloid angiopathy & $0.0(0.0)$ & $0.5(0.8)$ & $2.7(0.5)^{\star \star}$ \\
\hline Lacunar infarct & $0.0(0.0)$ & $2.5(0.9)^{\star \star}$ & $0.2(0.5)$ \\
\hline Territorial infarct & $0.0(0.0)$ & $2.0(1.4)^{\star \star}$ & $0.1(0.4)$ \\
\hline Haematoma & $0.0(0.0)$ & $0.9(1.2)$ & $0.4(0.7)$ \\
\hline Cortical microinfarcts & $0.2(0.4)$ & $2.2(1.3)^{\star \star}$ & $3.4(1.0)^{\star \star}$ \\
\hline Cortical microbleeds & $0.3(0.5)$ & $1.7(0.9)^{\star \star}$ & $2.0(1.3)^{\star \star}$ \\
\hline
\end{tabular}

${ }^{*} p \leq 0.01 ;{ }^{* *} p \leq 0.001$ 
The same findings were observed on the postmortem MRI, concerning WMCs, LIs, CoMBs and CoMIs (Table III).

On mutual comparison of the $\mathrm{VaD}$ and the MixD brain CoMBs, predominated in the frontal lobe and in the cerebellum of the former, while increased in the temporal and the occipital lobes in the latter group (Fig. 1). Cortical microinfarcts predominated in the frontal lobe and the cerebellum of the $\mathrm{VaD}$ group, while increased in the occipital lobe of the MixD group (Fig. 2). White matter changes predominated only in the temporal lobe of the MixD group (Fig. 3). As to LIs they were significantly increased in the corona radiata and the putamen in the $\mathrm{VaD}$ group (Fig. 4, Table IV).

\section{Discussion}

The present study shows a difference in the distribution and types of the small cerebrovascular lesions in patients with VaD compared to those with MixD. Their heterogeneity was already previously suspected $[16,22,25]$. Our previous neuropathological study demonstrated that LIs due to arteriosclerotic angiopathy are the most common lesions in VaD, while CAA related lesions are more frequent in MixD, suggesting that the latter represent the natural end-stage evolution of Alzheimer's disease [9]. There is a strong correlation between CAA and age $[17,19]$. However, in contrast to a previous neuropathological study [1], more territorial infarcts are observed in $\mathrm{VaD}$ than in MixD brains. This is probably due to more additional large-vessel disease in the former group [18].

Cortical microinfarcts predominate in the frontal lobe and in the cerebellum of $\mathrm{VaD}$ as cerebral arteriosclerosis is their main cause $[8,18]$, while according to the validated Boston criteria for CAA, they predominate in the occipital lobe in MixD [10]. The same is also observed for CoMBs, although also

Table III. Mean values (standard deviations) of the MRI lesions in normal controls (C) compared to those in vascular dementia (VaD) and mixed dementia (MixD) brains.

\begin{tabular}{|cccc|}
\hline MRI lesions & $\mathrm{C}$ & VaD & MixD \\
\cline { 2 - 4 } & $(n=11)$ & $(n=14)$ & $(n=24)$ \\
\hline White matter changes & $0.2(0.4)$ & $1.4(0.6)^{\star \star}$ & $1.5(0.6)^{\star \star}$ \\
\hline Lacunar infarct & $2.8(1.0)$ & $14.4(1.9)^{\star *}$ & $3.3(2.2)$ \\
\hline Cortical microinfarcts & $0.2(0.4)$ & $5.2(1.4)^{\star \star}$ & $4.9(0.8)^{\star \star}$ \\
\hline Cortical microbleeds & $3.3(1.1)$ & $10.4(1.6)^{\star \star}$ & $9.8(2.0)^{\star *}$ \\
\hline
\end{tabular}

${ }^{*} p \leq 0.01 ;{ }^{* *} p \leq 0.00$
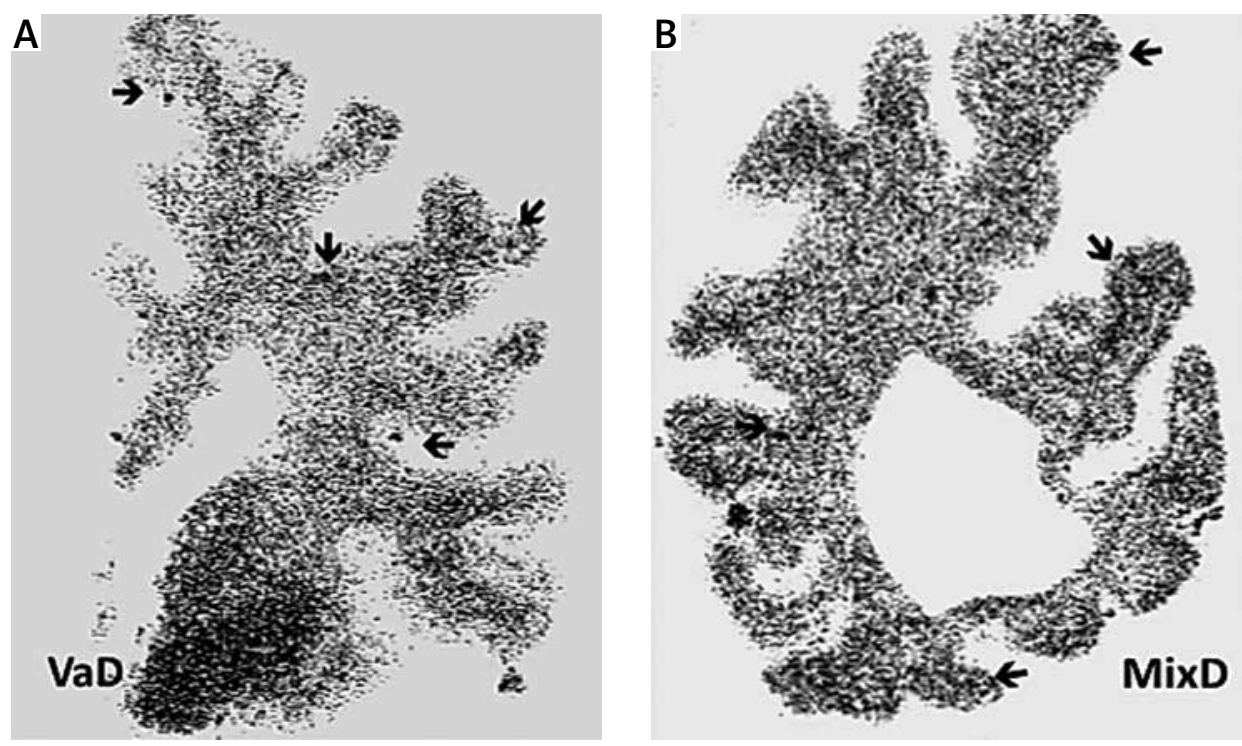

Fig. 1. T2* MRI demonstrating the presence of cortical microbleeds (arrows) on a coronal section of the frontal lobe in a patient with vascular dementia (A) and of the occipital lobe in a patient with mixed dementia (B). 

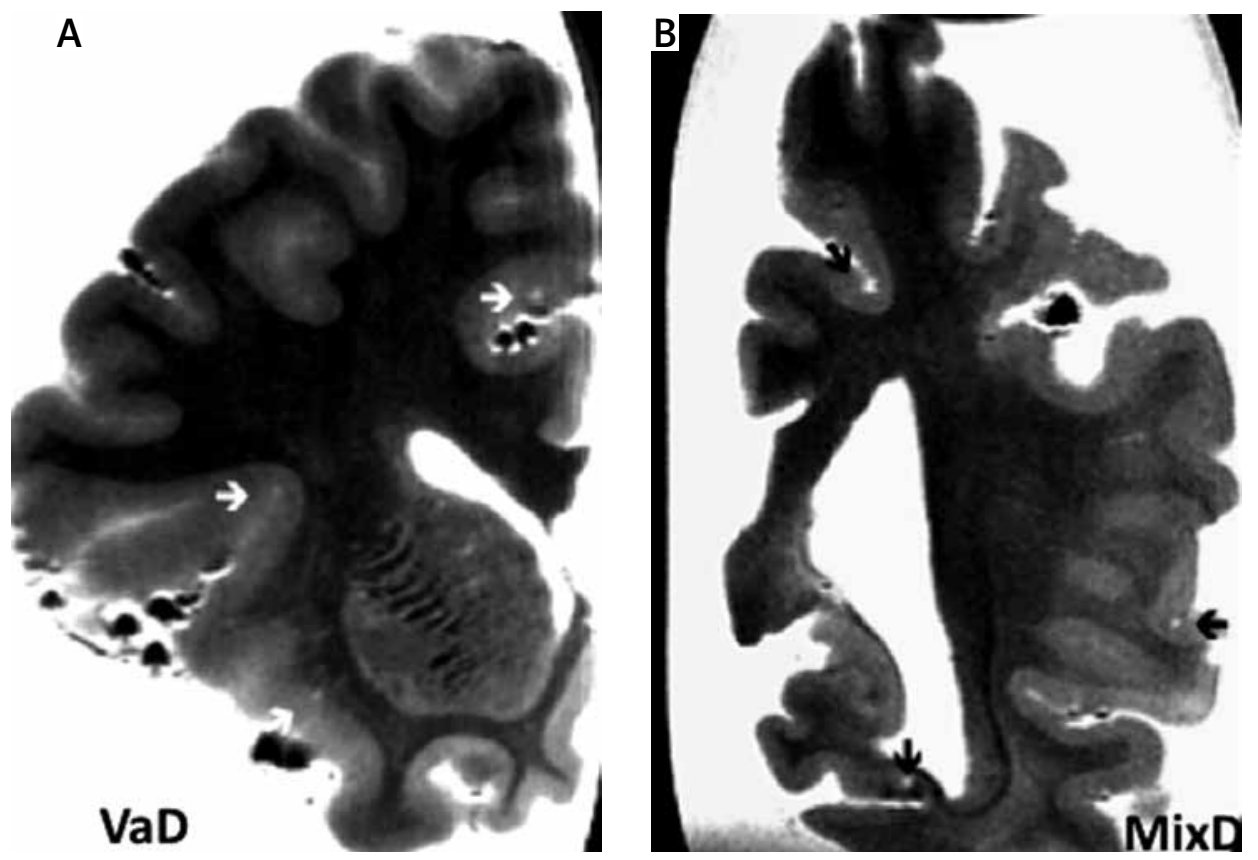

Fig. 2. T2 MRI demonstrating the presence of cortical microinfarcts (arrows) on a coronal section of the frontal lobe in a patient with vascular dementia (A) and of the occipital lobe in a patient with mixed dementia (B).
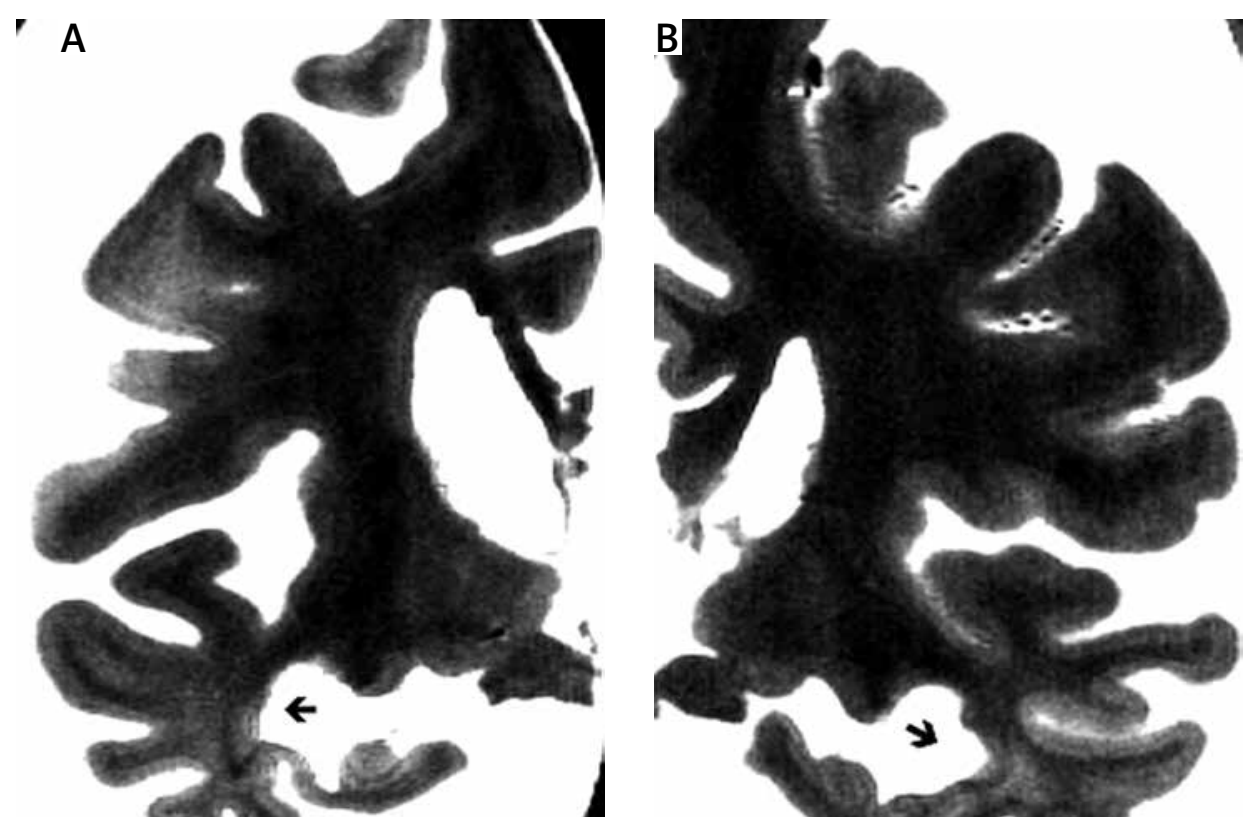

Fig. 3. T2 MRI of a central coronal section of a mixed dementia brain showing, in addition to the enlargement of the temporal horn, more selective temporal white matter changes.

highly present in the temporal lobe of MixD brains. The latter can be explained by the fact that CoMBs are not only due to micro-vascular lesions, but also to the severity of the neurodegenerative changes themselves in $A D$ [6].
White matter changes are, as a whole, as severe in $\mathrm{VaD}$ as in MixD [9]. The present study shows a predominance in the temporal lobe of MixD brains associated to the temporal lobe atrophy, due to the underlying severity of the Alzheimer lesions [3]. 
Table IV. Comparison of the mean values (standard deviations) of the topography of the small cerebrovascular lesions on magnetic resonance imaging between brains with vascular dementia (VaD) and mixed dementia (MixD)

\begin{tabular}{|c|c|c|}
\hline $\begin{array}{c}\text { Cerebrovascular } \\
\text { lesions }\end{array}$ & $\begin{array}{c}\mathrm{VaD} \\
(n=14)\end{array}$ & $\begin{array}{c}\text { MixD } \\
(n=24)\end{array}$ \\
\hline \multicolumn{3}{|l|}{ Cortical microbleeds } \\
\hline Frontal lobe & $4.1(0.8)^{\star \star}$ & $2.1(1.0)$ \\
\hline Temporal lobe & $1.5(0.9)$ & $2.6(1.0)^{\star \star}$ \\
\hline Parietal lobe & $1.4(0.6)$ & $1.6(1.1)$ \\
\hline Occipital lobe & $1.5(0.9)$ & $3.4(1.0)^{\star \star}$ \\
\hline Cerebellum & $2.7(1.1)^{\star \star}$ & $0.8(1.1)$ \\
\hline \multicolumn{3}{|l|}{ Cortical microinfarcts } \\
\hline \begin{tabular}{|l|} 
Frontal lobe \\
\end{tabular} & $1.6(0.8)^{\star}$ & $0.8(0.8)$ \\
\hline Temporal lobe & $0.4(0.6)$ & $0.9(0.8)$ \\
\hline Parietal lobe & $1.3(1.1)$ & $1.6(0.6)$ \\
\hline Occipital lobe & $0.5(0.3)$ & $1.4(0.8)^{\star \star}$ \\
\hline Cerebellum & $1.9(0.7)^{\star \star}$ & $0.4(0.6)$ \\
\hline \multicolumn{3}{|l|}{ White matter changes } \\
\hline Frontal lobe & $1.6(0.6)$ & $1.1(0.8)$ \\
\hline Temporal lobe & $0.3(0.6)$ & $1.3(0.9)^{\star \star}$ \\
\hline Parietal lobe & $0.9(0.8)$ & $1.7(0.9)$ \\
\hline Occipital lobe & $0.9(0.9)$ & $1.3(0.9)$ \\
\hline Cerebellum & $0.8(0.8)$ & $0.3(0.7)$ \\
\hline \multicolumn{3}{|l|}{ Lacunar infarcts } \\
\hline Corona radiata & $7.0(0.5)^{\star \star}$ & $0.6(0.8)$ \\
\hline Caudate nucleus & $1.1(0.8)$ & $0.4(0.7)$ \\
\hline Internal capsule & $1.1(0.6)$ & $0.6(0.8)$ \\
\hline Putamen & $3.2(0.8)^{\star \star}$ & $0.9(0.9)$ \\
\hline Globus pallidus & $1.3(0.9)$ & $0.3(0.4)$ \\
\hline Thalamus & $0.7(0.7)$ & $0.3(0.4)$ \\
\hline Cerebellum & $0.2(0.4)$ & $0.0(0.0)$ \\
\hline
\end{tabular}

${ }^{*} p \leq 0.01 ;{ }^{* *} p \leq 0.001$

Although in our previous study, only LIs in the corona radiata were found in $\mathrm{VaD}$ compared to MixD [12], our present study also shows an additional increase in the putamen. Their topography corresponds to the vascular territory of the lenticulostriate arteries [5]. These findings correlate well with "in vivo" measurements of lenticulostriate arteries using 7-tesla MRI that show fewer side-branches in $\operatorname{vaD}[24]$.

The present post-mortem MRI study shows clear differences in the distribution and the types of cerebrovascular lesions, confirming that $\mathrm{VaD}$ and MixD are different diseases.
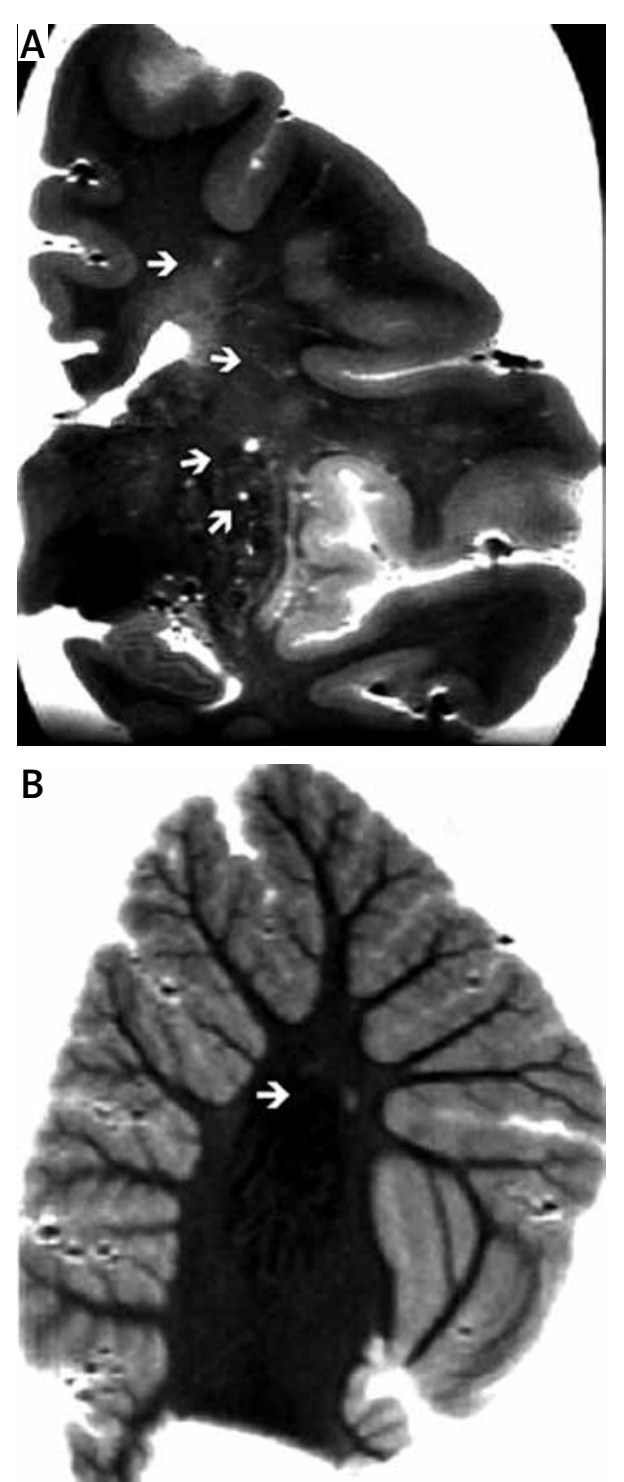

Fig. 4. T2 MRI showing on a central coronal section in addition to diffuse white matter changes also several lacunar infarcts in the corona radiata and putamen (arrows) in a patient with vascular dementia (A). A lacunar infarct is also observed in the cerebellar white matter of the same patient (B).

\section{Disclosure}

Authors report no conflict of interest.

\section{References}

1. Attems J, Jellinger KA. The overlap between vascular disease and Alzheimer's disease - lessons from pathology. BMC Med 2014; 12: 206. 
2. Braak H, Braak E. Evolution of neuronal changes in the course of Alzheimer's disease. Acta Neuropathol 1991; 82: 239-259.

3. Bombois S, Debette S, Bruander A, Delbeuck X, Delmaire C, Leys D, Pasquier F. Vascular subcortical hyperintensities predict conversion to vascular and mixed dementia in MCl patients. Stroke 2008; 39: 2046-2051.

4. Deramecourt V, Slade JY, Oakley AE, Perry RH, Ince PG, Maurage CA, Kalaria RH. Staging and natural history of cerebrovasular pathology in dementia. Neurology 2012; 78: 1043-1050.

5. De Reuck J. The human periventricular arterial blood supply and the anatomy of cerebral infarctions. Eur Neurol 1971; 5: 321-334.

6. De Reuck J, Deramecourt V, Cordonnier C, Leys D, Pasquier F, Maurage CA. Prevalence of small cerebral bleeds in patients with a neurodegenerative dementia: A neuropathological study. J Neurol Sci 2011; 300: 63-66.

7. De Reuck J, Auger F, Cordonnier C, Deramecourt V, Durieux N, Pasquier F, Bordet R, Maurage CA, Leys D. Comparison of 7.0-T $\mathrm{T}^{*}$-magnetic resonance imaging of cerebral bleeds in post-mortem brain sections of Alzheimer patients with their neuropathological correlates. Cerebrovasc Dis 2011; 31: 511-517.

8. De Reuck JL, Deramecourt V, Durieux N, Cordonnier C, Devos D, Defebvre L, Moreau C, Capparos-Lefebvre D, Pasquier F, Leys D, Maurage CA, Bordet R. The significance of cortical cerebellar microbleeds and microinfarcts in neurodegenerative and cerebrovascular diseases. A post-mortem 7.0-tesla magnetic resonance study with neuropathological correlates. Cerebrovasc Dis 2015; 39: 138-143.

9. De Reuck J, Deramecourt V, Cordonnier C, Pasquier F, Leys D, Maurage CA, Bordet R. Incidence of post-mortem neurodegenerative and cerebrovascular pathology in mixed dementia. J Neurol Sci 2016; 366: 164-166.

10. De Reuck j, Cordonnier C, Deramecourt V, Auger F, Durieux N, Leys D, Pasquier F, Maurage CA. Lobar intracerebral haematoma. Neuropathological and 7.0-tesla magnetic resonance imaging evaluation. J Neurol Sci 2016; 369: 121-125.

11. De Reuck J, Auger F, Durieux N, Cordonnier C, Deramecourt V, Pasquier F, Maurage CA, Leys D, Bordet R. The topography of cortical microinfarcts in neurodegenerative diseases and vascular dementia: a postmortem 7.0-tesla magnetic resonance imaging study. Eur Neurol 2016; 76: 57-61.

12. De Reuck J, Auger F, Durieux N, Cordonnier C, Deramecourt V, Pasquier F, Maurage CA, Leys D, Bordet R. Topographic distribution of white matter changes and lacunar infarcts in neurodegenerative and vascular dementia syndromes: A post-mortem 7.0-tesla magnetic resonance imaging study. Eur Stroke J 2016; 1: 122-129.

13. Ellis RJ, Olichney JM, Thal LJ, Mirra SS, Morris JC, Beekly D, Heyman A. Cerebral amyloid angiopathy in the brains of patients with Alzheimer's disease: the CERAD experience, Part XV. Neurology 1996; 46: 1592-1596.

14. Grinberg LT, Heinsen H. Toward a pathological definition of vascular dementia. J Neurol Sci 2010; 299: 136-138.

15. Jellinger KA, Attems J. Neuropathological evaluation of mixed dementia. J Neurol Sci 2007; 25: 80-87.

16. Jellinger KA. The pathology of "vascular dementia": a critical update. J Alzheimers Dis 2008; 14: 107-123.
17. Jellinger KA, Attems J. Prevalence and pathology of vascular dementia in the oldest-old. J Alzheimers Dis 2010; 21: 1283-1293.

18. Kitagawa K, Miwa K, Yagita Y, Okazaki S, Sakaguchi M, Mochizu$\mathrm{ki} \mathrm{H}$. Association between carotid stenosis or lacunar infarction and incident dementia in patients with vascular risk factors. Eur J Neurol 2015; 22: 187-192.

19. Kovari E, Herrmann FR, Hof PR, Bouras C. The relation between cerebral amyloid angiopathy and cortical microinfarcts in brain ageing and Alzheimer's disease. Neuropathol Appl Neurobiol 2013; 39: 498-509.

20. Niemantsverdriet E, Feyen BF, Le Bastard N, Martin JJ, Goeman J, De Deyn PP, Engelborghs S. Overdiagnosing vascular dementia using structural brain imaging for dementia work-up. J Alzheimers Dis 2015; 45: 1039-1043.

21. McAleese KE, Firbank M, Hunter D, Sun L, Hall R, Near JW, Mann DM, Esiri M, Jellinger KA, O’Brien JT, Attems J. Magnetic resonance imaging of fixed post mortem brains reliably reflects subcortical vascular pathology of frontal, parietal and occipital white matter. Neuropathol Appl Neurobiol 2013; 39: 485-497.

22. Lo RY, Jagust WJ; Alzheimer's Disease Neuroimaging Initiative. Vascular burden and Alzheimer disease pathological progression. Neurology 2012; 79: 1349-1355.

23. Lopez OL, Kuller LH, Becker JT, Jagust WJ, DeKosky ST, Fitzpatrick A, Breitner J, Lyketsos C, Kawas C, Carlson M. Classification of vascular dementia in the Cardiovascular Health Study Cognition Study. Neurology 2005; 64:1539-1547.

24. Seo SW, Kang CK, Kim SH, Yoon DS, Liao W, Worz S, Rohr K, Kim YB, Na DL, Cho ZH. Measurements of lenticulostriate arteries using $7 T$ MRI: new imaging markers for subcortical vascular dementia. J Neurol Sci 2012; 322: 200-205.

25. Staekenborg SS, van Straaten EC, van der Flier WM, Lane R, Barkhof F, Scheltens P. Small vessel versus large vessel vascular dementia: risk factors and MRI findings. J Neurol 2008; 255:1644-1651.

26. Trattnig S, Bogner W, Gruber S, Szomolanyi P, Juras V, Robinson S, Zbyn S, Haneder S. Clinical applications at ultrahigh field (7 T). Where does it make the difference? NMR Biomed 2015; 29: 1316-1334

27. Wang X, Valdés Hernandez MD, Doubal F, Chappell FM, Piper RJ, Deary IJ, Wardlaw JM. Development and initial evaluation of a semi-automatic approach to assess perivascular spaces on conventional magnetic resonance images. J Neurosci Methods 2015; 257: 34-44.

28. Yassi N, Desmond PM, Masters CL. Magnetic resonance imaging of vascular contributions to cognitive impairment and dementia. J Mol Neurosci 2016; 60: 349-353. 